\title{
Estudio sobre la lixiviación de cadmio, cromo y plomo de un limpiador lingual en saliva artificial
}

Study on the leaching of cadmium, chromium and lead from a tongue cleaner onto artificial saliva

Enrique Plaza ${ }^{1, a}$, Andrea Yipmantín ${ }^{2, b}$, Cristina Ikeda-Artacho ${ }^{3, e}$, Carlos Aldana ${ }^{3, d}$, Víctor HuanambalTiravanti ${ }^{3, c}$, Jaime Otero ${ }^{4, d}$, Fredy Gutiérrez-Ventura ${ }^{3, c}$.

\section{RESUMEN}

Objetivos: Determinar la presencia y concentración de los metales plomo, cadmio y cromo migrados desde el limpiador lingual "Alitest" en saliva artificial, y estimar la exposición oral a estas sustancias. Material y métodos: Diseño pre-experimental y analítico, para cuantificar las sustancias que son liberadas desde el limpiador lingual al contacto con saliva artificial. Los metales fueron analizados por AAS. Se estimaron los valores de exposición por ingesta oral que fueron comparados con valores de ingesta diaria tolerable para cada grupo etario. Resultados: Se cuantificó la migración de cadmio y cromo en concentraciones promedio de $0.008 \mathrm{mg} / \mathrm{L}$ y 0.437 $\mathrm{mg} / \mathrm{L}$, respectivamente. La concentraciòn de plomo estuvo por debajo de los niveles detectables utilizando el mètodo. A partir de estos valores se estimó la ingesta oral para cada grupo etario y se comparó con el valor provisional de ingesta mensual tolerable (PTMI) o Dosis de Referencia (RfD). Conclusiones: Las sustancias se cuantificaron en concentraciones normales, que no excedieron los valores máximos recomendados.

PALABRAS CLAVE: Cadmio, cromo, plomo, lixiviación, limpiador lingual.

Facultad de Estomatología, Universidad Peruana Cayetano Heredia. Lima, Perú.

Escuela de Estomatología Universidad Científica del Sur. Lima, Perú.

a. MSc. en Ciencias

b. Mg. en Química

c. Magister en Estomatología

d. Especialista en Ortodoncia

e. Doctor en Estomatología 


\section{SUMMARY}

Objectives: To determine the concentration of heavy metals cadmium and chromium leached from the tongue cleaner "Alitest" into artificial saliva and to assess oral exposure of these substances. Material and methods: Analytical pre-experimental design, to determine the amount of those substances released from the tongue cleaner onto artificial saliva and to quantified them by AAS. Assessment of exposure from oral intake values and comparison with the Provisional Tolerable Monthly Intake (PTMI) or Reference Dose (RfD) values. Results: Cadmium and chromium leachated into artificial saliva were quantified in concentrations of $0.008 \mathrm{mg} / \mathrm{L}$ and $0.437 \mathrm{mg} / \mathrm{L}$, respectively. Lead concentration was under detectable levels using the method. These values were used to estimate the oral intake for each age group and were compared to PTMI or RfD values. Conclusions: The substances were found in normal concentrations, none exceeding the maximum values of PTMI or RfD.

\section{KEYWORDS: Cadmium, chromium, lead, leaching, tongue cleaner.}

\section{INTRODUCCIÓN}

La lixiviación de sustancias químicas en la cavidad bucal debido a tratamientos odontológicos o por utilización de dispositivos de higiene bucal es un tema de gran relevancia, ya que representa una ruta de exposición que puede ocasionar toxicidad, enfermedades sistémicas, cáncer y mortalidad.

Se ha estudiado la migración de metales como mercurio, cobre y zinc en saliva artificial con el fin de determinar la liberación de estas sustancias en la cavidad bucal después de la aplicación de amalgamas dentales (1). Así también se ha evaluado la liberación de cobre y plomo producto de la utilización de prótesis dentales (2). Algunos estudios han concluido que los compuestos lixiviados derivados de BPA como las resinas dentales pueden inducir efectos adversos locales y sistémicos $(3,4)$.

Los dispositivos de higiene bucal están compuestos principalmente de termoplásticos, pigmentos, colorantes y aditivos como ftalatos, como es el caso de los cepillos dentales. Svendsen (5) evaluó la migración de sustancias químicas desde cepillos dentales, determinando que sustancias como 2-butoxietanol, 2-butoxietilacetato, ftalato de benzilbutilo y níquel pueden liberarse al ser utilizados. Análogamente, los dispositivos de limpieza lingual utilizados para reducir la halitosis, también pueden ser sujetos a este tipo de evaluaciones debido a que presentan componentes plásticos, colorantes y pigmentos que entran en contacto con la cavidad bucal. Sin embargo, luego de realizar una revisión de la literatura científica no hemos identificado estudios relacionados a dispositivos de higiene lingual.
Con el fin de estimar la exposición oral del uso diario de un limpiador lingual compuesto por poliestireno y colorantes orgánicos, se cuantificó la lixiviación de cadmio y cromo en saliva artificial en condiciones análogas a las fisiológicas, y se compararon los resultados de ingesta oral obtenidos con el valor provisional de ingesta mensual tolerable (PTMI) para el caso del cadmio y, la dosis de referencia (RfD), en el caso del cromo $(6,7)$.

\section{MATERIAL Y MÉTODOS}

Se realizó una investigación de tipo analítica con diseño pre-experimental.

\section{Procedimiento}

Se llevó a cabo la lixiviación de los metales pesados colocando 10 muestras de limpiador lingual, compuestos por poliestireno y colorantes orgánicos, en saliva artificial por un lapso de 1 hora a condiciones de temperatura de $37^{\circ} \mathrm{C}$. Luego se procedió a cuantificar los niveles de cadmio y cromo, migrados a la solución de saliva artificial, empleando espectrofotometría de Absorción Atómica Perkin Elmer Analyst 100 (AAS). A partir de esta cuantificación, se estimó la ingesta oral por el uso del dispositivo en la cavidad bucal, aplicando la siguiente ecuación (1):

Para la aplicación de esta fórmula se consideraron las siguientes variables:

$C_{\text {oral }}$ : Concentración de la sustancia migrada del producto por hora

$F_{\text {oral }}$ : Fracción absorbida (Biodisponibilidad)

$T_{\text {exposición }}$ : Tiempo en minutos

$N_{\text {eventos }}:$ Número de veces por día

MC: Masa corporal

$I_{\text {oral }}$ Ingesta de la sustancia 
Para efectos del estudio, se asumió un tiempo de exposición $\left(T_{\text {exposición }}\right)$ por la utilización del dispositivo para higiene lingual de 2 minutos (para 2 usos diarios de 1 minuto cada uno). El peso del sujeto se estableció de acuerdo al percentil 50 de la tabla de peso y talla (8). Se estimó la absorción oral específica para cada sustancia asumiendo los valores de biodisponibilidad máximos publicados en la literatura. En el caso del cadmio se encontró que el límite inferior y superior es de $9,4 \%$ y $83,3 \%$, respectivamente. Para el caso del cromo, los valores límites son $0,5 \%$ y $2,0 \%$, respectivamente (9-10). Luego de efectuar el cálculo de ingesta oral, se procedió a contrastar los resultados obtenidos con los valores de ingesta tolerable (tabla 1).

\section{RESULTADOS}

La medición de las sustancias lixiviadas desde el limpiador lingual hacia la saliva artificial se cuantificó en $0,8 \mu \mathrm{g} / \mathrm{unidad}$ y $43,6 \mu \mathrm{g} / \mathrm{unidad}$, para el cadmio y cromo, respectivamente. El nivel de plomo en la muestra estuvo por debajo del límite de detección del método L.D. $=0,1 \mathrm{mg} / \mathrm{L}$.

Se extrajeron los valores de peso y talla correspondientes al percentil 50 para cada uno de los años de edad

Tabla 1. Datos de los límites máximos de Ingesta Diaria Tolerable $(6,7)$.

\begin{tabular}{cccc}
\hline & \multicolumn{2}{c}{ Ingesta tolerable - PTMI ( $\mu \mathrm{g} / \mathrm{kg}$-mes $)$ o RfD ( $\mu \mathrm{g} / \mathrm{kg}$-día $)$} \\
Metal & Niños (3-8 años) & $\begin{array}{c}\text { Preadolescentes y } \\
\text { Adolescentes }(9-17 \text { años })\end{array}$ & Adultos $(18+)$ \\
\hline Cadmio (PTMI) & 25 & 25 & 25 \\
Cromo (VI) (RfD) & 3 & 3 & 3 \\
\hline
\end{tabular}

Tabla 2. Resultados de la ingesta oral en diferentes grupos etarios.

\begin{tabular}{lcccc}
\hline & Edad & Peso & \multicolumn{2}{c}{ Ingestión oral } \\
Grupo etario & $($ años $)$ & promedio $(\mathrm{kg})$ & Cadmio & Cromo \\
& $(8)$ & $(8)$ & $\mu$ g/kg-mes & $\mu$ g/kg-día \\
\hline Niños & 5 & 18 & 0,036 & 0,0016 \\
& 6 & 21 & 0,033 & 0,0014 \\
Preadolescentes y & 7 & 23 & 0,030 & 0,0013 \\
adolescentes & 8 & 26 & 0,027 & 0,0011 \\
& 10 & 29 & 0,024 & 0,0010 \\
& 11 & 32 & 0,021 & 0,0009 \\
& 12 & 36 & 0,018 & 0,0008 \\
& 13 & 41 & 0,015 & 0,0007 \\
& 14 & 46 & 0,015 & 0,0006 \\
& 15 & 52 & 0,012 & 0,0006 \\
& 16 & 57 & 0,012 & 0,0005 \\
& 17 & 61 & 0,012 & 0,0005 \\
& 18 & 65 & 0,009 & 0,0004 \\
& & 67 & 0,009 & 0,0004 \\
& & & &
\end{tabular}


entre los 5 y 18 años. Utilizando estos datos y los resultados de la medición de sustancias migradas cuantificadas se calcularon los valores de ingestión oral para cada uno de los metales pesados en estudio (tabla 2).

\section{DISCUSIÓN}

La absorción oral de las sustancias depende de la cantidad lixiviada, tipo de metal, especiación y propiedades químicas. La absorción a través de la cavidad bucal es específica para cada sustancia y se han empleado valores de biodisponibilidad (9-10) de cada metal cuantificado por espectrofotometría después del contacto del limpiador lingual con saliva artificial, cuyos resultados aparecen en la tabla 2. La concentración de plomo no pudo ser determinada por encontrarse debajo del límite de cuantificación del método empleado. A partir de las concentraciones de cadmio y cromo se halló la ingesta oral diaria, empleando los valores del límite superior de biodisponibilidad para asumir la exposición máxima por cada sustancia.

Con base en la concentración de los metales detectados en la muestra y en las condiciones de exposición estipuladas, se estima que ninguna de las sustancias migradas se encontró en concentraciones que excedan los valores máximos de PTMI o RfD para cada caso. Dado que las estimaciones se han realizado considerando un individuo de 5 años de edad de $18 \mathrm{~kg}$ de peso, así como una absorción máxima de las sustancias en análisis, los resultados del presente estudio sugieren que el limpiador lingual "Alitest" tiene un adecuado margen de seguridad para estos metales.

\section{Correspondencia:}

Enrique Plaza

Correo electrónico: eplazav@hotmail.com

\section{REFERENCIAS BIBLIOGRÁFICAS}

1. Saghiri MA, Banava S, Sabzian MA, et al. Correlation between long-term in vivo amalgam restorations and the presence of heavy elements in the dental pulp. J Trace Elem Med Biol. 2014;28:200-204.

2. Manan N, Harun W, Shri D, Ghani S, Kurniawan T, Ismail M, Ibrahim M. Study of corrosion in biocompatible metals for implants: A review. Journal of Alloys and Compounds. 2017;701: 698-715.

3. Madhyastha P. Naik D, Kotian R, Padma D, Srikant N, Bhat K. Evaluation of cytotoxicity of silorane and methacrylate based dental composites using gingival fibroblasts. J Clin. Diagn Res. 2015;9(1): ZC05ZC08. doi: $10.7860 / J C D R / 2015 / 10269.5366$

4. Kramer AG, Vuthiganon J, Lassiter CS. Bis-GMA affects craniofacial development in zebrafish embryos (Danio rerio). Environ Toxicol Pharmacol. 2016;43:159-165.

5. Svendsen N, Pedersen S. Survey of chemical substances in consumer products no. 42, 2004; Survey of chemical substances in toothbrushes. Warszawa: Danish Technological Institute; 2004.

6. Mueller U, Agudo A, Akesson A, et al. Safety evaluation of certain food additives and contaminants. Ginebra: WHO Food Additives Series: 64. Ginebra: WHO 2011.p. 305-380.

7. U.S. Environmental Protection Agency National Center for Environmental Assessment Integrated Risk Information Systems (IRIS) Chemical Assessment Summary on Chromium (VI). U.S. Environmental Protection Agency National Center for Environmental Assessment; 2017.p. 1-33.

8. National Center for Health Statistics. Grow charts - Weight for age percentiles. Washington: National Center for Health Statistics; National Center for Chronic Disease Prevention and Health Promotion; 2000.

9. Ollson C, Smith E, Herde P, Juhas Z Influence of sample matrix on the bioavailability of arsenic, cadmium and lead during co-contaminant exposure. Sci Tot Environ. 2017; 595: 660-665.

10. Zhao D, Li J, Li C, et al. Lead relative bioavailability in lip products and their potential health risk to women. Environ Sci Technol, 2016; 50(11):6036-43.
Recibido: 19-02-2017

Aceptado: 23-05-2017 